\title{
Führung im Public Sector - Wie wird geführt und welche Veränderungen stehen an?
}

\author{
Dominik Vogel \\ ORCID: 0000-0002-0145-7956
}

This is the accepted manuscript of an article published by Haufe Verlag in PERSONALquarterly. The article is available at https://www.haufe.de/personal/zeitschrift/personalquarterly/personalquarterly-12017-digitale-transformation-personalquar-

terly_48_396138.html

Suggested citation:

Vogel, D. (2017). Führung im Public Sector: Wie wird geführt und welche Veränderungen stehen an? PERSONALquarterly, 69(1), 15-19. 


\section{Abstract}

Forschungsfrage: Was bedeutet Führung im öffentlichen Sektor, wie wird dort geführt und wie unterscheidet sich der öffentliche Sektor vom Privatsektor?

Methodik: Schriftliche Befragung von 64 Führungskräften und 464 Mitarbeiterinnen und Mitarbeitern der öffentlichen Verwaltung.

Praktische Implikationen: Führung im öffentlichen Sektor unterscheidet sich deutlich von Führung im Privatsektor. Die Ergebnisse zeigen, dass Führung im öffentlichen Sektor weiter gestärkt werden muss und Führungskräfteauswahl und -entwicklung sowie Feedbackinstrumente verbessert werden sollten.

\section{Summary}

Research question: What is public leadership, how do public managers lead and how does the public sector differ from the private sector?

Methodology: Survey of 64 managers and 464 subordinates in the public sector.

Practical implications: Leadership in the public sector differs from the private sector. The results show that leadership in the public sector has to be strengthened and leader selection, leadership development and feedback instruments have to be improved. 
Im Gegensatz zum Privatsektor hat Führung im öffentlichen Sektor lange Zeit kaum eine Rolle gespielt. Dies mag nicht zuletzt daran liegen, dass das bürokratische Ideal eigentlich darauf abzielt, Institutionen und Abläufe zu schaffen, innerhalb derer der einzelne Mitarbeiter grundsätzlich austauschbar ist. Was zunächst einen befremdlichen Eindruck hinterlässt, hat einen begrüßenswerten Hintergrund. Verwaltung sollte so gestaltet sein, dass es für jede Bürgerin und jeden Bürger unerheblich ist, wer das eigene Anliegen bearbeitet. Jeder hat dieselben Rechte und Pflichten und wird entsprechend der rechtlichen Vorgaben behandelt.

Aus diesem Ideal hat man lange Zeit abgeleitet, dass es auch innerhalb des öffentlichen Sektors unerheblich ist, wer eine Führungsfunktion wahrnimmt, sofern die fachliche Eignung vorliegt. Die Führungskraft hat dementsprechend die Verantwortung, für die Erledigung der Aufgaben und die Einhaltung der rechtlichen und organisatorischen Vorgaben zu sorgen. Eine darüberhinausgehende Verantwortung, die einem modernen Verständnis von Führung gerecht wird, ist im bürokratischen Idealbild demnach nicht vorgesehen.

Dieses Idealbild hat zwar in der Praxis nicht in der reinen Form Anwendung gefunden und es gab auch immer wieder Behörden, die versuchten, dieses Bild zu durchbrechen. Dennoch bestimmte es lange Zeit den Blick auf das Thema Führung. Bei der Besetzung von Führungspositionen hat man hauptsächlich auf die fachlichen Fähigkeiten der Bewerber geachtet und wenig darauf, ob ein Bewerber in der Lage sein wird, eine Organisationseinheit auch tatsächlich zu führen. Man könnte daher spöttisch sagen, der beste Sachbearbeiter wurde zum Obersachbearbeiter befördert. Auch die Führungskräfteentwicklung spielte lange Zeit abseits der Vermittlung dienst- und arbeitsrechtlicher Grundlagen kaum eine Rolle.

In den vergangenen Jahren ist hier allerdings ein Wandel zu beobachten. Dieser resultiert nicht zuletzt aus einem Wertewandel der Belegschaft, wie ihn auch Unternehmen der Privatwirtschaft zu spüren bekommen, und einer zunehmenden Arbeitsverdichtung und Veränderungsintensität, die die Bedeutung von Führung massiv verstärkt haben. Erste Verwaltungen haben damit begonnen, gezielt auf die Führungsfähigkeiten von Nachwuchskräften zu achten und diese auch bei den vorhandenen Führungskräften zu fördern. Als Beispiel kann hier die Bundesagentur für Arbeit mit ihrem umfassenden Führungskräfteentwicklungsprogramm „In Führung gehen“ (Bundesagentur für Arbeit 2009) genannt werden.

Mit dem geringen Interesse der Praxis am Thema Führung ging auch ein geringes Interesse der Forschung einher. Dies führt dazu, dass wir bis heute wenig darüber wissen, wie im öffentlichen Sektor geführt wird. Dies trifft nicht nur für Deutschland, sondern auf die Public-Management-Forschung insgesamt zu. Erst das auflebende Interesse einzelner Forscher in den letzten fünf Jahren fördert zunehmend empirische Erkenntnisse zu Tage. Eine dieser Studien wurde vom Autor dieses Beitrags durchgeführt (Vogel 2016) und soll im Folgenden näher vorgestellt werden. Dabei steht die Frage im Vordergrund, wie im öffentlichen Sektor geführt wird und welche Erkenntnisse davon abgeleitet werden können. 


\section{Was ist eigentlich Führung im Public Sector?}

An dieser Stelle bietet sich ein kurzer Einschub an, der erläutern soll, was Führung im öffentlichen Sektor eigentlich ist. Dies ist wichtig, da die Antwort darauf noch komplexer ist als sie es für den Privatsektor ohnehin schon ist. Man kann Führung im öffentlichen Sektor in drei Teilaspekte untergliedern (s. Abbildung 1).

Administrative Leadership: Dieser Bereich bildet den Schwerpunkt dieses Beitrags. Administrative Führung deckt die Führung von Organisationen und Organisationseinheiten des öffentlichen Sektors durch ernannte Führungskräfte ab. Davon ausgenommen ist folglich Führung durch direkt oder indirekt gewählte Führungskräfte wie Minister oder Bürgermeister. Diese fallen in die nachfolgende Kategorie.

Political Leadership: Dieser Teil deckt Führung durch gewählte Führungskräfte ab und hat einen Bezug über die eigentliche Verwaltung hinaus. Dazu zählt auch Führung im Rahmen von Politikgestaltung.

Community Leadership: Dieser Teilaspekt umfasst die Führung und Gestaltung von Gemeinwesen und insbesondere der (sektorübergreifenden) Kooperation verschiedener Akteure. Hierzu gehört insbesondere auch die Führung von Netzwerken.

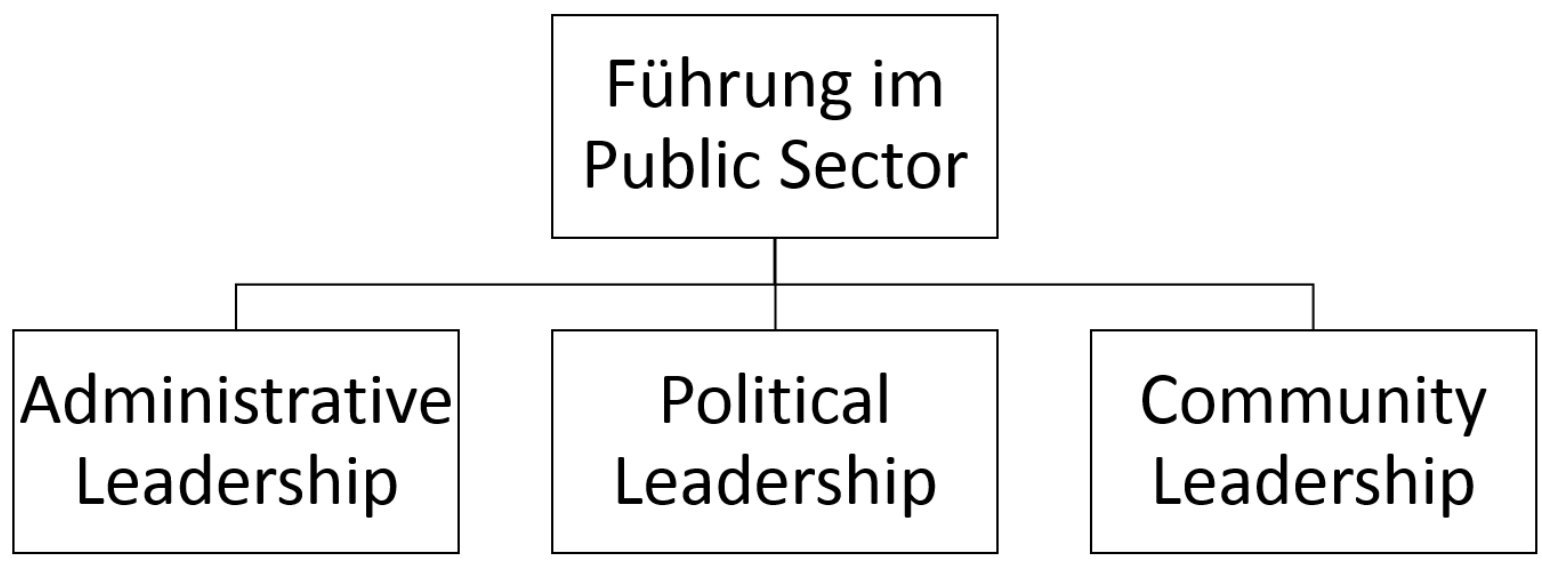

Abbildung 1: Formen von Führung im Public Sector. Quelle: Eigene Darstellung nach Van Wart 2013: 527 


\section{Wie wird in der öffentlichen Verwaltung geführt?}

Konzentrieren wir also auf den Bereich der administrativen Führung, also jenen Bereich, der in der Regel auch dann betrachtet wird, wenn wir von Führung in der Privatwirtschaft sprechen. Wie wird im öffentlichen Sektor geführt? Ist Führung in der Verwaltung etwas Anderes als in der Privatwirtschaft?

Hierzu soll nachfolgend ein breiter Blick auf Führung geworfen werden. Hierzu bietet sich die "Taxonomy of Effective Leadership Behavior" von Yukl et al. (2002) an. Diese teilen Führung in vier Bereiche: Aufgaben-, Beziehungs-, Veränderungs- und Außenorientierung. Der obere Teil von Tabelle 1 gibt hierzu eine kurze Zusammenfassung.

Tabelle 1: Taxonomy of Effective Leadership Behavior nach Yukl. et al. 2002 und eigene Ergänzungen

\section{Dimension von Führungs- verhalten}

Aufgabenorientierung

Beziehungsorientierung

Außenorientierung

Ethikorientierung

Sachbearbeitungsorientierung

\section{Beschreibung}

Alle Führungstätigkeiten, die sich auf die Erledigung der Aufgaben konzentrieren (planen, kontrollieren, Zuständigkeiten klären, ...)

Alle Führungsaktivitäten, die sich auf die Geführten richten (unterstützen, weiterentwickeln, Leistung anerkennen, ...)

Veränderungsorientierung

Alle Führungsaktivitäten, die sich auf die Initiierung und Umsetzung von Wandel konzentrieren (Vision entwickeln, für Veränderung eintreten, ...)

Alle Führungstätigkeit, die über die eigene Organisation(seinheit) hinausgeht (netzwerken, repräsentieren, ...)

Alle Führungstätigkeiten, die auf die Vermittlung und Durchsetzung ethischer Standards zielen (kommunizieren, sanktionieren, ...)

Alle Führungstätigkeiten, die auf die eigene Expertenrolle gerichtet sind (schwierige Aufgaben erledigen, Informationen beschaffen, ...) 
Neben diesen allgemein gebräuchlichen Dimensionen von Führung, wurde die Taxonomie um zwei weitere Dimensionen ergänzt, die den besonderen Charakter des öffentlichen Sektors widerspiegeln. Dies ist zunächst die Ethikorientierung. Diese besondere Form von Führung hat auch in der privatwirtschaftlichen Diskussion in den letzten ca. fünfzehn Jahren eine zunehmende Aufmerksamkeit erfahren. Für den öffentlichen Sektor ist sie jedoch von besonderer Bedeutung. Die öffentliche Aufmerksamkeit gegenüber Verwaltungen, das engmaschige Regelwerk und die besondere Werthaltung innerhalb des öffentlichen Sektors stellen besondere Anforderungen an Führungskräfte. Als zweites wurde mit der Sachbearbeitungsorientierung eine Dimension ergänzt, die charakteristisch für Führung im öffentlichen Sektor ist. Insbesondere von Führungskräften unterer Hierarchieebenen wird ein großer Anteil eigener Facharbeit erwartet. Sie sollen Experten auf ihrem Gebiet sein und entsprechende Aufgaben übernehmen.

\section{Empirische Ergebnisse zu Führung im Public Sector}

Die Taxonomy von Yukl et al. (2002) bildete die Basis für eine Befragung von unteren Führungskräften und deren Geführten in drei Behörden. An der Umfrage haben 64 Führungskräfte und 464 Mitarbeiterinnen und Mitarbeiter teilgenommen (Rücklaufquote: 57,1 \% bzw. 34 \%).

Die Teilnehmerinnen und Teilnehmer der Befragung wurden jeweils gefragt, wie stark sie einzelnen Aussagen zu ihrem Führungsverhalten, beziehungsweise dem der eigenen Führungskraft zustimmen. Die Antwortmöglichkeiten reichten dabei von 1 (stimme überhaupt nicht zu) bis 5 (stimme voll und ganz zu). Abbildung 2 fasst die Ergebnisse der Befragung zusammen. Die Punkte zeigen dabei an, wie die Geführten ihre Führungskräfte im Durchschnitt einschätzen. Die Rauten geben die durchschnittliche Selbsteinschätzung der Führungskräfte wieder. Die Fehlerbalken um die einzelnen Punkte und Rauten geben den 95 \%-Konfidenzintervall wieder.

Es zeigt sich, dass das Führungsverhalten aus Sicht der Geführten eher mittelmäßig ausgeprägt ist und noch ein gewisses Verbesserungspotential bietet. Die Werte liegen im Mittel zwischen 3,0 und 3,5. Die geringste Ausprägung beobachten die Geführten bei der Veränderungsorientierung. Dies spiegelt relativ deutlich eine grundlegende Kritik am öffentlichen Sektor wieder, der zu wenig Wert auf Veränderungen lege. Die übrigen Dimensionen von Führung liegen aus Sicht der Geführten mit 3,3 bis 3,5 etwa gleich auf. Ein deutlich anderes Bild ergibt die Selbsteinschätzung der Führungskräfte. Hier liegen die Mittelwerte mit 3,8 bis 4,5 deutlich höher. Zudem schätzen die Führungskräfte ihre Beziehungsorientierung deutlich intensiver ein als die anderen Dimensionen. Auch die Ethikorientierung sticht mit einem Mittelwert von 4,2 hervor.

Besonders auffällig sind beim Blick auf Abbildung 2, wie bereits erwähnt, die Differenzen zwischen der Selbsteinschätzung der Führungskräfte und der Einschätzung der Geführten. Die Differenzen betragen zwischen 0,4 und 1,0 Punkten. Auf einer Skala von 1 bis 5 entspricht dies einer Differenz 
von $10 \%$ bis $25 \%$. Diese deutliche Differenz wurde in anderen Studien bisher kaum beobachtet. Ein gewisses $M a ß$ an Selbstüberschätzung scheint zwar überall vorhanden zu sein, aber so groß wie hier sind sie nur in wenigen Fällen. Interessant ist dabei, dass die wenigen weiteren Studien, die solche Differenzen zwischen Selbst- und Fremdwahrnehmung beobachteten, ebenfalls aus dem öffentlichen Sektor stammen. Die Vermutung liegt daher nahe, dass dies eine Besonderheit des öffentlichen Sektors ist und einer eingehenderen Untersuchung bedarf.

Eine Ursache für die großen Differenzen zwischen Selbst- und Fremdeinschätzung im öffentlichen Sektor könnte sein, dass Führung insgesamt wenig thematisiert wird und insbesondere institutionalisierte Feedbackinstrumente, wie $360^{\circ}$-Feedbacks, kaum Anwendung finden und die Führungskräfte daher kaum Rückmeldung über ihr eigenes Führungsverhalten erhalten.

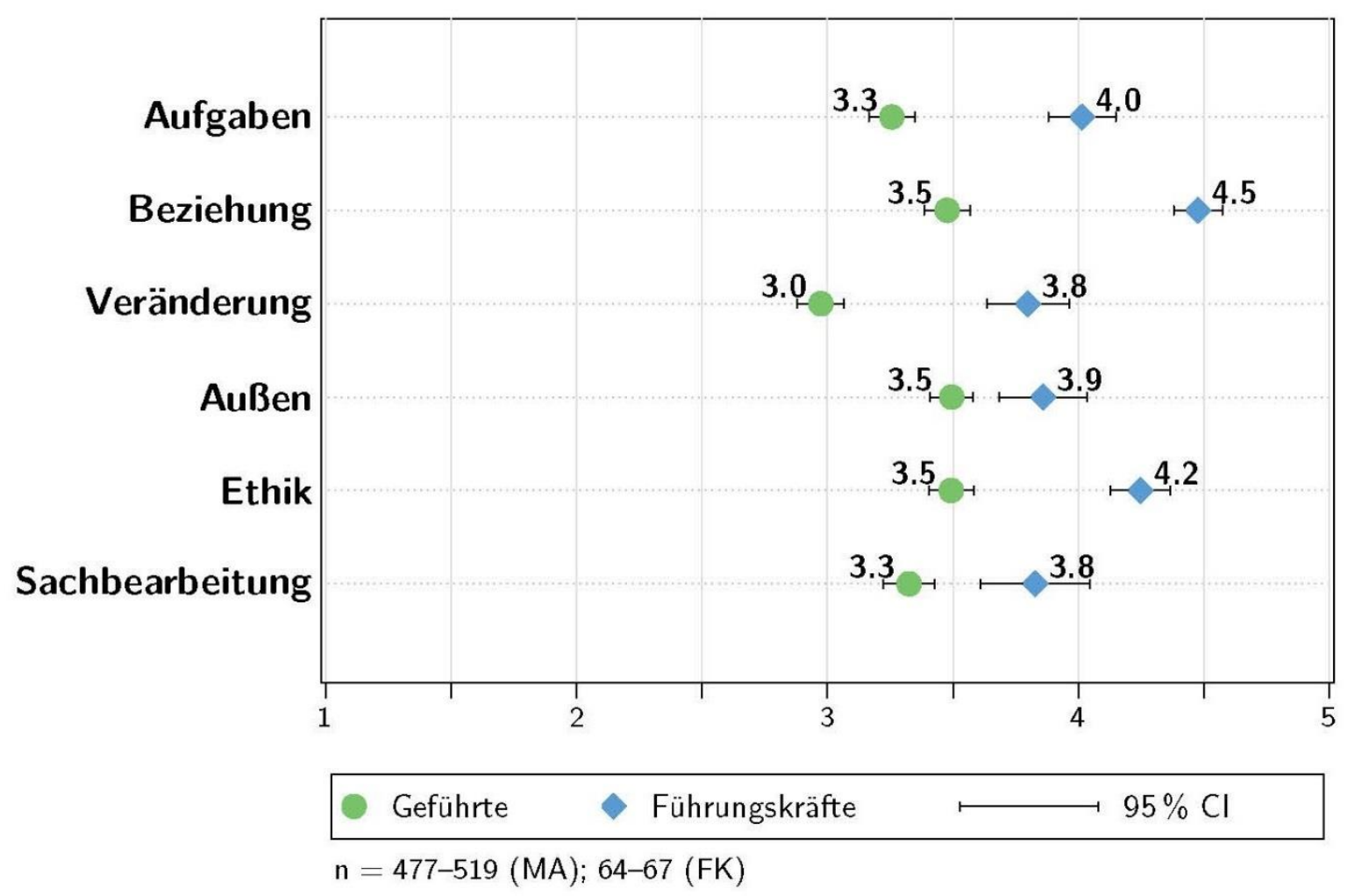

Abbildung 2: Führung im Public Sector. Einschätzung von Führungsverhalten durch Führungskräfte und Geführte. Quelle: Eigene Darstellung 


\section{Besondere Aspekte von Führung im öffentlichen Sektor}

Die aufgeführten Ergebnisse beinhalten bereits zwei Besonderheiten von Führung im öffentlichen Sektor. Dies ist zunächst die Ethikorientierung. Aus Sicht der Geführten zeigen die Führungskräfte dieses Führungsverhalten in selber Intensität wie Beziehungs- oder Außenorientierung - den Kernaspekten von Führung. Die Führungskräfte, sehen sich bei der Ethikorientierung selbst besonders aktiv.

Auch die Sachbearbeitungsorientierung stellt eine Besonderheit des öffentlichen Sektors dar. Wie die Ethikorientierung so nimmt die eigene fachliche Arbeit ein ähnliches Ausmaß an, wie klassische Führungsaufgaben. An dieser Stelle sind sich Geführte und Führungskräfte in ihrer Einschätzung auch verhältnismäßig einig.

Andere Studien haben der Besonderheit von Führung im öffentlichen Sektor noch stärkere Aufmerksamkeit gewidmet. Hierbei sticht vor allem jene von Tummers und Knies (2016) hervor. Diese entwickelten vier Rollen die Führungskräfte im öffentlichen Sektor erfüllen müssen und die diese deutlich von Führungskräften der Privatwirtschaft abgrenzen (s. Tabelle 2).

Tabelle 2: Rollen von Führungskräften im öffentlichen Sektor. Nach Tummers und Knies 2016, S. 436

\section{Rolle}

Accountability Leadership

Rule-Following Leadership

Political Loyalty Leadership

Network Governance Leadership

\section{Beschreibung}

Die Führungskraft ermutigt die Geführten, ihre Arbeit gegenüber Stakeholdern zu erläutern und zu rechtfertigen.

Die Führungskraft ermutigt die Geführten, in Übereinstimmung mit rechtlichen Vorgaben zu handeln.

Die Führungskraft motiviert die Geführten, ihre Arbeit mit den Interessen politischer Leitungskräfte in Einklang zu bringen auch wenn dies für sie zusätzlichen Aufwand verursacht.

Die Führungskraft ermutigt die Geführten, relevante Stakeholder aktiv miteinander zu vernetzen.

Hier zeigt sich deutlich, welche weiteren Aufgaben Führungskräften im öffentlichen Sektor zukommt und wie sich deren Arbeit abseits klassischer Führungsaufgaben von der Arbeit im Privatsektor unterscheidet. 


\section{Schlussfolgerungen und praktische Implikationen}

Welche Schlussfolgerungen können nun aus den bisherigen Ausführungen gezogen werden? Vier Punkte sollen an dieser Stelle herausgegriffen werden.

Führung in Public Sector ist etwas Anderes als Führung im Privatsektor. Die grundlegenden Verantwortlichkeiten sind sicherlich in beiden Sektor ähnlich. Aufgaben müssen verteilt und kontrolliert werden, Mitarbeiter müssen motiviert werden, Veränderungen müssen initiiert und vorangetrieben werden und Organisationen müssen repräsentiert werden. Darüber hinaus unterscheiden sich die Rahmenbedingungen innerhalb derer dies stattfindet stark. Dies führt auch dazu, dass Führungskräften Aufgaben zufallen, die spezifisch für den öffentlichen Sektor sind. Accountability, rechtliche Vorgaben, politische Interessen und Stakeholder spielen eine wesentlich größere Rolle und stellen besondere Herausforderungen an Führungskräfte des öffentlichen Sektors.

Führung ist im öffentlichen Sektor ausbaufähig. Die Bedeutung, die Führung beigemessen wird ändert sich zwar in letzter Zeit, dieser Wandel schreitet jedoch relativ langsam voran. Dies schlägt sich auch im Führungsverhalten nieder. Die präsentierten Ergebnisse zeigen, dass aus Sicht der Geführten in allen Dimensionen Verbesserungspotential vorhanden ist. Damit verbunden ist auch, dass Führungskräfteauswahl eine stärkere Rolle einnehmen muss. Bisher ist es in vielen Bereichen üblich, bei der Auswahl auf fachliche Fähigkeiten zu fokussieren. Führungsfähigkeiten spielen hingegen eine geringe Rolle. Dasselbe trifft auf Führungskräfteentwicklung zu. In diesem bedeutsamen Feld wird kaum strategisch agiert und es bleibt oft den einzelnen Führungskräften überlassen, sich um entsprechende Fortbildungsmaßnahmen zu kümmern. Hier könnte wesentlich mehr getan werden.

Institutionalisierte Feedbackinstrumente müssen etabliert werden. Die großen Differenzen zwischen Selbst- und Fremdwahrnehmung zeigen, dass beim Thema Führung zu wenig kommuniziert wird. Ohne geeignete Instrumente fällt es Führungskräften schwer, ein realistisches Bild von ihrem Führungsverhalten zu gewinnen. Dies könnte beispielsweise durch regelmäßige $360^{\circ}$-Feedbacks verbessert werden. 


\section{Literaturverzeichnis}

Bundesagentur für Arbeit (Hrsg.) (2009): In Führung gehen. Initiative zur Stärkung der Führungsfähigkeit der Teamleiter/innen. Bundesagentur für Arbeit: Nürnberg.

Tummers, L. G.; Knies, E. (2016): Measuring Public Leadership. Developing Scales for Four Key Public Leadership Roles. In: Public Administration, Jg. 94, H. 2, S. 433-451.

Van Wart, M. (2013): Administrative Leadership Theory: A Reassessment After 10 Years. In: Public Administration Jg. 91, H. 3, S. 521-543.

Vogel, D. (2016): Führung im öffentlichen Sektor - Eine empirische Untersuchung von Einflussfaktoren auf das Führungsverhalten. Universitätsverlag Potsdam: Potsdam.

Yukl, G. A.; Gordon, A.; Taber, T. (2002): A Hierarchical Taxonomy of Leadership Behavior: Integrating a Half Century of Behavior Research. In: Journal of Leadership \& Organizational Studies, Jg. 9, H. 1, S. 1532. 